\title{
Top quark pair properties (spin correlations, charge asymmetry and complex final states) with the ATLAS detector
}

\author{
Liza MIJOVIC* ${ }^{* \dagger}$ \\ CEA-Saclay \\ E-mail: liza.mijovicecern.ch
}

In proton-proton collisions at the Large Hadron Collider, pairs of top and antitop quarks are expected to be mostly produced through gluon fusion, in contrast to production at the Tevatron, where quark annihilation dominates. The ATLAS collaboration has used the relatively large number of top-antitop quark events to measure the spin correlation between top and antitop quarks as well as the top quark charge asymmetry. These constitute important tests of QCD and are sensitive to new physics. We also discuss top quark pair production in association with the Standard Model electroweak gauge bosons.

The European Physical Society Conference on High Energy Physics

18-24 July, 2013

Stockholm, Sweden

\footnotetext{
* Speaker.

$\dagger$ on behalf of the ATLAS Collaboration. The author acknowledges funding by the LabEx P2IO in the framework Investissements d'Avenir (ANR-11-IDEX-0003-01) managed by the French National Research Agency (ANR).
} 


\section{Introduction}

We review the following top-antitop quark pair $(t \bar{t})$ properties measured with the ATLAS detector [1]: measurement of spin correlation (Section 2), charge asymmetry (Section 3) and $t \bar{t}$ production in association with the Standard Model (SM) electroweak (EWK) gauge bosons $(t \bar{t}+Z, t \bar{t}+\gamma$, Section 4). The up-to-date documentation of all public ATLAS top quark measurements is available at [2].

The measurements are done with the large number of $t \bar{t}$ pairs in the dataset produced by the $7 \mathrm{TeV} p p$ collisions in the Large Hadron Collider (LHC). Many of the first studies of top quark pairs have been performed by experiments at the Fermilab Tevatron Collider. The differences between the $t \bar{t}$ dataset collected at the Tevatron and the LHC, most relevant for the reviewed results, are as follows.

- The LHC experiments have collected a notably larger sample of $t \bar{t}$ events than the Tevatron experiments. The LHC dataset collected at $\sqrt{s}=7 \mathrm{TeV}$ has approximately half the integrated luminosity ( $\mathscr{L}_{\text {int }}$ ) of the $\sqrt{s}=1.96 \mathrm{TeV}$ Tevatron dataset. However, the $t \bar{t}$ production crosssection $\left(\sigma_{t \bar{t}}\right)$ at the LHC is approximately 25 times larger for $7 \mathrm{TeV}$ than the $\sigma_{t \bar{t}}$ at the Tevatron [4]. Thus at the LHC, more precise measurements as well as measurements of processes that have too small cross-section to be measured at the Tevatron can be performed, such as associated $t \bar{t}+Z$ production.

- The dominant $t \bar{t}$ production mechanism at the LHC is $g g$ fusion, while in collisions produced at the Tevatron the $q \bar{q}$ annihilation dominates [3].

- While the LHC collides proton-proton ( $p p$ ) beams the Tevatron collisions are from protonantiproton $(p \bar{p})$ beams.

The difference in the dominant production mechanism and the $p p$ rather than $p \bar{p}$ collisions are of particular importance for the measurements of the asymmetries expected in $t \bar{t}$ production.

\section{Measurement of spin correlation in $t \bar{t}$ events}

Spin correlation in $t \bar{t}$ production has been measured with the data collected at $\sqrt{s}=7 \mathrm{TeV}$ and integrated luminosity of $\mathscr{L}_{\text {int }}=2.05 \mathrm{fb}^{-1}$ [5]. In the SM, the spins of the $t$ and $\bar{t}$ quarks in $t \bar{t}$ pairs produced at the LHC are expected to be correlated. Since the top quark decays before hadronising, its spin information is transferred to the decay products. The measurement is performed using the events corresponding to the dileptonic (ee, $e \mu$ or $\mu \mu$ ) decay channel topology, with two opposite sign high- $\mathrm{p}_{\mathrm{T}}$ leptons, large missing transverse energy $\left(\mathrm{E}_{\mathrm{T}}\right)$ and at least two jets. Spin correlation information is extracted from the distribution of the azimuthal angle difference between the leptons $(\Delta \phi)$ shown in Fig. 1 (left). In the figure the data as well as distributions corresponding to the SM prediction (solid line) and no-spin-correlation hypothesis (dashed line) are shown.

The analysis strategy is to construct $\Delta \phi$ distributions for different fractions of events with SM-like spin correlation $\left(f_{S M}\right)$. The parameter $f_{S M}$, defined so that $f_{S M}=1$ is the SM result, is estimated from a binned likelihood fit to the data where the $\Delta \phi$ distributions vary with respect to $f_{S M}$ in the fit. The backgrounds to $t \bar{t}$ production are estimated using Monte Carlo (MC) predictions 

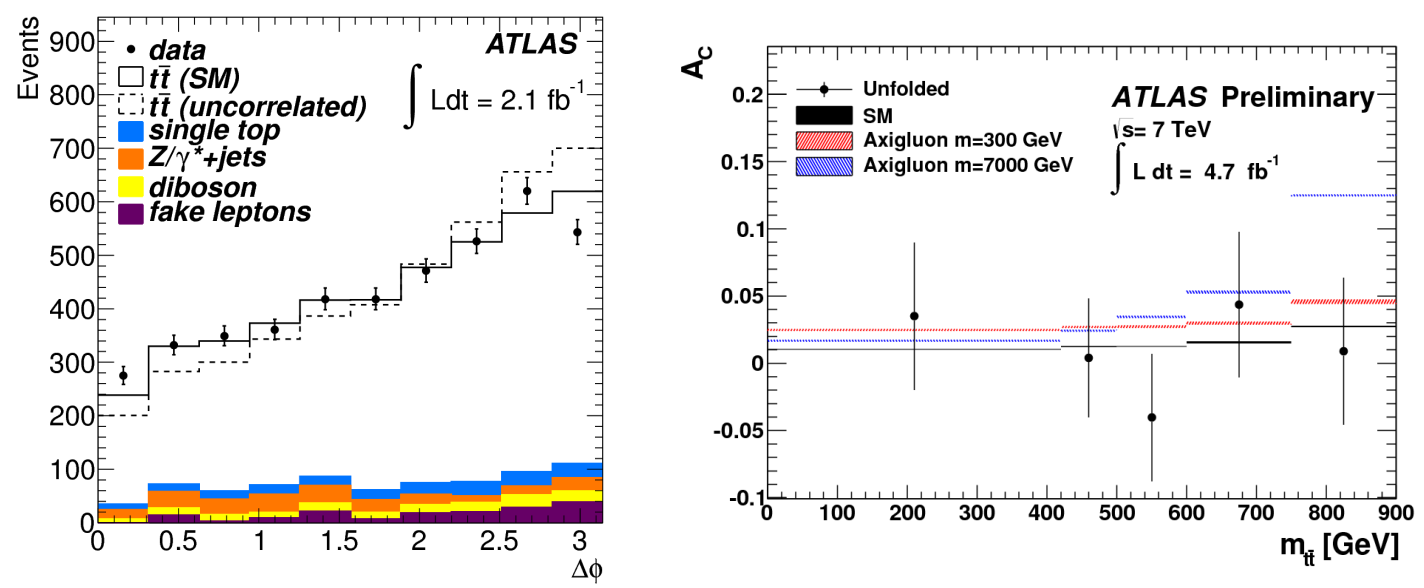

Figure 1: Left: lepton $\Delta \phi$ distribution used for the measurement of the spin correlation in $t \bar{t}$ production [5]. Right: $t \bar{t}$ charge asymmetry for several bins of $t \bar{t}$ pair invariant mass [6].

normalized to the theoretically predicted cross-sections. The exceptions are the Z+jets dielectron and dimuon processes, for which the normalization is extracted from the data and the "fake lepton backgrounds, which are estimated from the data. The fitted value $f_{S M}=1.30 \pm 0.14$ stat. ${ }_{-0.22}^{+0.27}$ syst. is consistent with the SM prediction. The no-spin-correlation hypothesis is excluded with 5.1 $\sigma$ statistical significance. The systematic uncertainty exceeds the statistical uncertainty for the measurement, with the largest sources being the jet energy scale, the jet energy resolution and the fake lepton background.

\section{Measurement of the charge asymmetry in $t \bar{t}$ events}

In SM $t \bar{t}$ production, the momentum of the $t(\bar{t})$ quark is expected to be correlated with the initial state $q(\bar{q})$ longitudinal momentum. The largest contribution to the correlation comes from the interference between the Born and loop diagrams in the $q \bar{q}$ initiated production [7]. The asymmetry in the momentum distributions of $q$ and $\bar{q}$ thus translates to an asymmetry in the rapidity $(y)$ of the $t \bar{t}$ system as follows.

- At the Tevatron Collider: the asymmetric collisions of the $p$ and $\bar{p}$ beams results in the forward-backward asymmetry $A_{F B}$ which is defined in terms of the number of events $N$ :

$$
A_{F B}=\frac{N(\Delta y>0)-N(\Delta y<0)}{N(\Delta y>0)+N(\Delta y<0)}, \Delta y=y_{t}-y_{\bar{t}} .
$$

- At the LHC: the SM effect causing the forward-backward asymmetry at the Tevatron is manifest in the charge asymmetry $A_{C}$ :

$$
A_{C}=\frac{N(\Delta|y|>0)-N(\Delta|y|<0)}{N(\Delta|y|>0)+N(\Delta|y|<0)}, \Delta|y|=\left|y_{t}\right|-\left|y_{\bar{t}}\right| .
$$

The Tevatron $A_{F B}$ measurements are significantly larger than the SM prediction [8, 9]. The datatheory difference is enhanced in some differential asymmetry distributions: for example, the asym- 
metry for events with $t \bar{t}$ invariant mass $m_{t \bar{t}}>450 \mathrm{GeV}$ bin is found to be $3.4 \sigma$ above the SM prediction in [8].

The ATLAS collaboration has measured the charge asymmetry in $t \bar{t}$ production using singlelepton [6] and dilepton [10] topologies at $\sqrt{s}=7 \mathrm{TeV}$ and integrated luminosity of $\mathscr{L}_{\text {int }}=4.7 \mathrm{fb}^{-1}$. The single-lepton channel measurement requires events with a high- $\mathrm{p}_{\mathrm{T}}$ isolated lepton $(e, \mu)$, large $\mathrm{Z}_{\mathrm{T}}$ and at least four jets, at least one of which is required to be tagged as $b$-jet. The backgrounds are estimated using the MC predictions normalized to the theoretically predicted cross-sections, except for the $W+$ jets and QCD multijet backgrounds. The $t$ and $\bar{t}$ momenta are reconstructed using a kinematic fit based on a likelihood approach. For the purpose of theory comparisons, the results are unfolded for the detector effects using a Bayesian unfolding procedure [11]. The measured inclusive asymmetry after the unfolding, $A_{C}=0.006 \pm 0.010$ ( stat. + syst.), is compatible with the SM prediction of $A_{C}=0.0123 \pm 0.0005$ [12]. The differential measurement of the $A_{C}$ as a function of the invariant mass of the $t \bar{t}$ system $\left(m_{t \bar{t}}\right)$ is shown in Fig. 1 (right). The asymmetry is also measured as a function of $t \bar{t}$ system transverse momentum and rapidity, as well as a function of $m_{t \bar{t}}$ after a requirement is made on the minimum $t \bar{t}$ system velocity. All the differential measurements are consistent with SM predictions. In this measurement, the statistical uncertainty dominates over the systematic uncertainty.

In the dilepton $(e e, e \mu, \mu \mu)$ channel the event selection requires that events have exactly two opposite-sign leptons $(e, \mu)$, large $\mathrm{B}_{\mathrm{T}}$ and at least two jets. The backgrounds are estimated using $\mathrm{MC}$ predictions normalized to the theoretically predicted cross-sections with the exception of the QCD background for which a data-driven estimate is used. The $t$ and $\bar{t}$ momenta are reconstructed using a maximum likelihood approach, based on the calculation of the leading order matrix-element. For the purpose of theory prediction comparisons, the results are corrected for acceptance and detector effects using correction factors. Apart from the $t \bar{t}$ asymmetry, the lepton based asymmetry (corresponding to $\Delta|y|=\left|y_{l+}\right|-\left|y_{l-}\right|$ in Eq. 3.2) is measured. The values of the $t \bar{t}$ and lepton asymmetries after the background subtraction and correction factors are $A_{C}^{t \bar{t}}=0.057 \pm 0.024$ stat. \pm 0.015 syst. and $A_{C}^{l l}=0.023 \pm 0.012$ stat. \pm 0.008 syst., respectively. Both values are consistent with the $\mathrm{SM}$ predictions $A_{C}^{t \bar{t}}=0.0123 \pm 0.0005$ and $A_{C}^{l l}=0.004 \pm 0.001$. For the measurement, the statistical uncertainty exceeds the systematic uncertainty. The dominant sources of systematic uncertainty are from QCD background modeling and signal $t \bar{t}$ modeling.

\section{Measurements of $t \bar{t}$ production in association with neutral EWK gauge bosons}

While the $t \bar{t}+Z$ production cross-section is too small to be detectable at the Tevatron, the direct measurements are for the first time feasible at the LHC. Various beyond-the-SM (BSM) theories with a strongly coupled Higgs sector predict a notably modified $t t Z$ coupling strength with respect to the SM predictions [13]. Thus, the $t \bar{t}+Z$ measurements are a sensitive probe of BSM theories. The ATLAS collaboration has searched for $t \bar{t}+Z$ production with the data collected at $\sqrt{s}=7 \mathrm{TeV}$ and integrated luminosity of $\mathscr{L}_{\text {int }}=4.7 \mathrm{fb}^{-1}$ [14]. The search is performed in the final states with three leptons, large $\mathrm{Z}_{\mathrm{T}}$ and four jets, at least one of which is required to be $b$-tagged. After the event selection cuts, the expected $t \bar{t}+Z$ signal is $0.85 \pm 0.04$ stat. \pm 0.14 syst. events. The number of background events with three leptons amongst the hard process decay products is estimated from the MC to be $0.28 \pm 0.05$ stat. \pm 0.14 syst. events. The background contribution 
due to the fake lepton candidates arising from mis-identified hadrons, leptons from heavy-flavour decay and photon conversions is estimated using a data-driven approach to be $0.0_{-0.0}^{+1.6}$ events. One data event is observed. A $95 \%$ confidence level upper limit to $t \bar{t}+Z$ production of $0.71 \mathrm{pb}$ is set, which is consistent with the SM prediction of $0.14 \mathrm{pb}$ [15]. The dominant source of systematics uncertainty comes from the background normalization.

The measurement of the $t \bar{t}+\gamma$ cross-section [16] has been performed with the data collected at $\sqrt{s}=7 \mathrm{TeV}$ and integrated luminosity of $\mathscr{L}_{\text {int }}=1.04 \mathrm{fb}^{-1}$. It uses the final states corresponding to the single-lepton $t \bar{t}$ decay topology with one high- $\mathrm{p}_{\mathrm{T}}$ lepton $(e, \mu)$, large $\mathrm{E}_{\mathrm{T}}$, at least four high$\mathrm{p}_{\mathrm{T}}$ jets, one or more of which are required to be $b$-tagged. Events are also required to contain at least one photon candidate with $\mathrm{p}_{\mathrm{T}}>15 \mathrm{GeV}$. After the event selection cuts 52(70) events are observed in the $e(\mu)$ channel. The sources of backgrounds come from processes containing prompt photons and fake photons (for example photons from $\pi^{0}$ decays). The separation of prompt and fake photons is based on the track isolation variable $\left(\mathrm{p}_{\mathrm{T}}^{\text {cone20 }}\right)$, which is defined as a sum of $\mathrm{p}_{\mathrm{T}}$ of all tracks within a cone of $R<0.2$ around the candidate photon. The $\mathrm{p}_{\mathrm{T}}^{\text {cone20 }}$ distributions for each of the prompt and fake photons are obtained from the $Z \rightarrow e e$ and the jet stream data, respectively. As shown in Fig. 2 (left), the $\mathrm{p}_{\mathrm{T}}^{\text {cone20 }}$ distributions of fake photons are expected to be notably wider than the prompt photon distributions. The fractions of prompt and fake photons are extracted from a fit, using these distributions as templates. The signal region $\mathrm{p}_{\mathrm{T}}^{\text {cone20 }}$ data distributions and the MC distributions after the fit are shown in Fig. 2 (right). The measured cross-section value for the
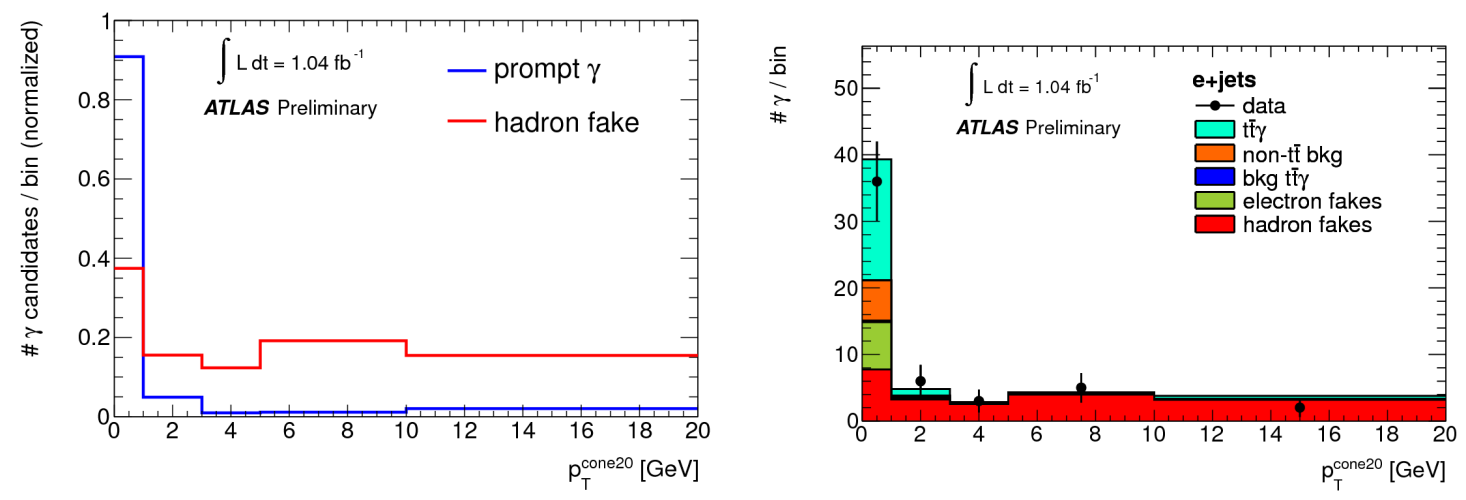

Figure 2: Photon track isolation variable $\mathrm{p}_{\mathrm{T}}^{\text {cone20 }}$ used in measurement of the $t \bar{t}+\gamma$ cross-section [16]. Left: $\mathrm{p}_{\mathrm{T}}^{\text {cone20 }}$ distributions for prompt and fake photons. Right: data and MC distributions of $\mathrm{p}_{\mathrm{T}}^{\text {cone20 }}$ in the signal region.

production of photons with $\mathrm{p}_{\mathrm{T}}>8 \mathrm{GeV}$ is $\sigma(t \bar{t} \gamma)=2.0 \pm 0.5$ stat. \pm 0.7 syst. \pm 0.08 lumi. pb. This is consistent with the SM prediction $\sigma(t \bar{t} \gamma)=2.1 \pm 0.4 \mathrm{pb}$. The dominant sources of systematics uncertainty for the measurement are from the jet energy scale, pile-up and signal modeling.

\section{Summary and Outlook}

We reviewed the following top quark pair properties measured with the ATLAS detector: measurement of spin correlation, charge asymmetry and $t \bar{t}$ production in association with the SM gauge bosons $(\gamma, Z)$ using $p p$ collisions collected at $\sqrt{s}=7 \mathrm{TeV}$. All results are consistent with the SM 
predictions. Many of the top quark properties measurements, for example the measurement of spin correlation and $t \bar{t}+\gamma$ measurements, are already limited by systematic rather than statistical uncertainty. Notable improvement of precision with the $8 \mathrm{TeV}$ and future LHC datasets therefore requires dedicated work to reduce the systematic uncertainties.

\section{References}

[1] ATLAS Collaboration, The ATLAS Experiment at the CERN Large Hadron Collider, JINST 3, (2008) S08003

[2] ATLAS Collaboration top group public results webpage:

https://twiki.cern.ch/twiki/bin/view/AtlasPublic/TopPublicResults

[3] CDF Collaboration, A. Abulencia et al., Measurement of the fraction of $t \bar{t}$ production via gluon-gluon fusion in $p \bar{p}$ collisions at $\sqrt{s}=1.96 \mathrm{TeV}$, Phys. Rev. D 79 (2009) 031101, ([arXiv: 0807 . 4262])

[4] M. Czakon, The total top quark pair production cross-section at hadron colliders through $O\left(\alpha_{S}^{4}\right)$, Phys. Rev. Lett. 110 (2013) 252004, ([arXiv: 1303.6254])

[5] ATLAS Collaboration, Observation of spin correlation in ttbar events from pp collisions at $\sqrt{s}=7 \mathrm{TeV}$ using the ATLAS detector, Phys. Rev. Lett. 108 (2012) 212001, ([arXiv:1203.4081])

[6] ATLAS Collaboration, Measurement of the top quark pair production charge asymmetry in proton-proton collisions at $\sqrt{s}=7 \mathrm{TeV}$ using the ATLAS detector, ATLAS-CONF-2013-078, https://cdsweb.cern.ch/record/1562978

[7] J. H. Kuhn and G. Rodrigo, Charge asymmetries of top quarks at hadron colliders revisited, JHEP 1201, (2012) 063, ([arXiv:1109.6830])

[8] CDF Collaboration, T. Aaltonen et al., Evidence for a Mass Dependent Forward-Backward Asymmetry in Top Quark Pair Production, Phys. Rev. D 83 (2011) 112003, ([arXiv: 1101.0034$]$ )

[9] D0 Collaboration, V. M. Abazov et al., Forward-backward asymmetry in top quark-antiquark production, Phys. Rev. D 84 (2011) 112005, ([arXiv: 1107.4995])

[10] ATLAS Collaboration, Measurement of the charge asymmetry in dileptonic decay of top quark pairs in pp collisions at $\sqrt{s}=7 \mathrm{TeV}$ using the ATLAS detector, ATLAS-CONF-2012-057,

https://cdsweb.cern.ch/record/1453785

[11] G. Choudalakis, Fully Bayesian Unfolding, [arXiv:1201.4612]

[12] W. Bernreuther and Z.-G. Si, Top quark and leptonic charge asymmetries for the Tevatron and LHC, Phys. Rev. D 86 (2012) 034026, ([arXiv:1205.6580])

[13] U. Baur et al., Probing Electroweak Top Quark Couplings at Hadron Colliders, Phys. Rev. D 71 (2005) 054013, ([arXiv: hep-ph / 0412021$])$

[14] ATLAS Collaboration, Search for $t \bar{t} Z$ production in the three lepton final state with $4.7 \mathrm{fb}^{-1}$ of $\sqrt{s}=7 \mathrm{TeV}$ pp collision data collected by the ATLAS detector, ATLAS-CONF-2012-126, http://cdsweb.cern.ch/record/1474643

[15] A. Kardos et al., Top quark pair production in association with a Z-boson at next-to-leading-order accuracy, Phys. Rev. D 85 (2012) 054015, ([arXiv: 1111.0610$]$ )

[16] ATLAS Collaboration, Measurement of the inclusive $t \bar{t} \gamma$ cross section with the ATLAS detector, ATLAS-CONF-2011-153, http://cdsweb. cern.ch/record/1398197 